\title{
Full title: Exclusive breastfeeding is more common among HIV-infected than HIV- uninfected Kenyan mothers at 6 weeks and 6 months postpartum
}

Running title: Maternal HIV status affects how infants are breastfed up to 6 months of age

Authors:

Shadrack Oiye ${ }^{1}$ BSc, MSc, PhD (Corresponding author)

Walter Mwanda ${ }^{1} \mathrm{MBCHB}, \mathrm{MSc}, \mathrm{PhD}$

Mary Mugambi ${ }^{2} \mathrm{BSc}, \mathrm{MSc}, \mathrm{PhD}$

Suzanne Filteau ${ }^{3}$ BSc, MSc, PhD

Victor Owino² BSc, MSc, PhD

${ }^{1}$ University of Nairobi Institute of Tropical and Infectious Diseases (UNITID). P.O Box

19676 - 00200 Nairobi, Kenya. Email: oiyeshad@gmail.com Telephone: +254-722-

759449

${ }^{2}$ Technical University of Kenya. P. O Box 52428 - 00200, Nairobi Kenya.

${ }^{3}$ London School of Hygiene and Tropical Medicine, Keppel Street, London, WC1E 7HT United Kingdom.

Word counts: 1) Abstract: 240 words 2) Text: 3,718 words......Max 2,948 (excluding references)

Number of tables: 3 . Number of figures: 1

Source of support: International Atomic Energy Agency (IAEA), The Nutricia Research Foundation and National Council for Science, Technology and Innovation (NACOSTI) Kenya. 


\begin{abstract}
Objective: To compare breastfeeding practices determined by mothers' own recall versus a stable isotope technique (deuterium oxide dilution) among HIV-infected and HIV-uninfected mothers at 6 weeks and 6 months postpartum.
\end{abstract}

Methods: Exclusive breastfeeding (EBF) rates were assessed cross-sectionally at 6 weeks and 6 months postpartum among $75 \mathrm{HIV}$-positive and $68 \mathrm{HIV}$-negative women attending postnatal care. EBF was derived from own 24-hour recall of foods that were fed to the infant and by objective measurement of non-human milk water intake using deuterium oxide (DO) dilution technique.

Results: Multivariable logistic analyses were adjusted for infant sex, gravidity, maternal age, marital status and maternal education. Using recall method, a greater proportion of HIV-infected mothers exclusively breastfed than HIV-uninfected mothers both at 6 weeks postpartum [94.1\% versus $76.9 \%$, respectively (aOR: 7.81; 95\% CI: 1.9-31.6, p=0.004)] and at 6 months postpartum [75\% versus 59.7\%, respectively (aOR: 2.27; 95\% CI: 1.0-5.3, p=0.058)]. At 6 weeks postpartum EBF rates from the DO technique were $23.5 \%$ and $13.8 \%$ for HIV-positive and HIVnegative mothers, respectively (aOR: 0.35; 95\% CI: 0.11-1.04, p=0.059). At 6 months postpartum, the DO technique determined EBF rates were $43.3 \%$ among HIV-positive and 24.2\% among HIV-negative mothers, respectively (aOR: 2.4; 95\% CI: 1.0-5.7, $\mathrm{p}=0.048$ ).

Conclusions: HIV-infected mothers are more likely to exclusively breastfeed compared to HIVuninfected mothers. In this resource poor setting, maternal recall overestimates EBF rates as compared to the deuterium oxide dilution technique. Validating EBF recall data using the objective DO technique is highly recommended for accurate tracking towards global targets on breastfeeding practices. 


\section{Introduction}

Due to the risk of transmission of the virus through breast milk, HIV infection has over the years presented a challenge on how resource-limited HIV-positive mothers should feed their infants. ${ }^{1-3}$ It is currently recommended that irrespective of maternal HIV status and that of the infant, mothers should exclusively breastfeed (EBF) until their infant are 6 months old while antiretroviral drugs are provided to the mother or infant. ${ }^{4} \mathrm{EBF}$ is protective against mortality and morbidity of HIV-exposed infants. ${ }^{4}$ However, there is limited data on how the current breastfeeding recommendation impacts breastfeeding practices of HIV-positive mothers. EBF rate is a key variable to measure in infant and young child feeding or nutrition surveys, and globally country-specific Demographic Health Surveys (DHS) collect the data every five years. EBF rates are responsive to policies, strategies and programs in support of EBF. ${ }^{5}$ These rates have been based on maternal recall of what was fed to an infant 24 hours preceding the interview. Maternal self-reported breastfeeding practices are based on recalls that are determined cross-sectionally and liable to memory errors as well as social desirability bias as mothers may report practices they have been told are sound. Since the recalls refer to only the past 24 hours and thus do not capture day-to-day variation, they are known to overestimate EBF rates. ${ }^{6,7}$ Therefore, an objective indicator of EBF is important for interpreting programmatic data including evaluating the effectiveness of breastfeeding policies and strategies. The deuterium oxide dilution (DO) dose-to-the-mother method is a stable isotope technique which can objectively assess breast milk volume and whether an infant has consumed non-breast milk fluids, an indication of non-exclusive breastfeeding. ${ }^{8}$ The DO technique assesses breast milk and non-human milk water intake data over a 14-day period and is useful for validation of the 
mother-reported EBF practice based on recall. The study aimed to compare EBF practice between HIV-positive versus HIV-negative mothers using maternal recall method and DO technique.

\section{Methods}

\section{Study design and setting}

This was part of a study designed to compare breast milk intake between HIV-uninfected infants whose mothers are HIV-1 infected (HIV-exposed, uninfected; HIV-EU) and infants whose mothers are HIV-1 negative (HIV-unexposed; HIV-U). EBF was derived from either mother's own 24 hour recall of what foods were fed to the infant and by objective measurement of nonhuman milk water intake using the DO technique. The study was based at the Maternal and Child Health (MCH) Clinic of Siaya County Referral Hospital in Western Kenya. Mothers were approached to enrol into the study as they came for their post-natal care between February 2014 and September 2014. Siaya County is a resource-poor area in the Lake Victoria Region of Kenya where HIV rates are among the highest in Kenya (15.9\% and $23.3 \%$ among men and women, respectively). ${ }^{9}$

\section{Recruitment of mothers}

Seventy five (75) HIV-positive and 68 HIV-negative mothers attending post-natal clinic for the first set of child vaccinations at 6 weeks postpartum were systematically sampled and recruited into the study and followed up at 6 months of age. Based on the $\mathrm{MCH}$ attendance register, it was estimated that approximately 10-20 mothers would attend the clinic daily at 6 weeks postpartum. The MCH was operational from Monday to Friday every week. To achieve the desired sample 
size, the study aimed to recruit 5-10 mothers-infant dyads per day. Therefore we approached every second mother in the queue at the clinic.

\section{Inclusion and exclusion criteria}

All HIV-infected and HIV-uninfected mothers were eligible for inclusion if their infants were HIV-uninfected at the time of enrolment at 6 weeks after birth. Mother-infants dyads of HIVuninfected infants aged 6 weeks (+/- 8 days) were eligible for this study. The following infants were excluded: infants having $<2500 \mathrm{~g}$ birth weight (from the mother-child clinic card), preterm infants, infants not able to breastfeed, infants or mothers showing signs of being very ill (not admitted and no plan for admission in the hospital). To minimise loss to follow-up, those intending to move away from Siaya District 7-10 months from the date of recruitment into the study were also excluded.

\section{Sample size}

The sample size was computed based on the objective of comparing breast milk intake of HIVEU and HIV-U infants. ${ }^{10} \mathrm{~A}$ mean difference in breast milk intake of $10 \mathrm{~g} / \mathrm{kg}$ body weight/day between HIV-EU and HIV-U infants and standard deviation of $18 \mathrm{~g} / \mathrm{kg}$ body weight/day were used in calculations. ${ }^{11}$ With a statistical power of $80 \%$ and $5 \%$ level of significance, a minimum sample size of 50 mother-infants dyads for each of the two age cohorts was required. It was expected that about 25 would be lost to follow-up (33\% attrition); this number was therefore added to the 50 to make a sample size of 75 per group. 


\section{Maternal and infant HIV testing}

Maternal HIV status at 6 weeks and 6 months after birth was determined by antibody testing by Colloidal Gold, (KHB Shanghai Kehua Bioengineering Co. Ltd). At pregnancy, the mothers had been tested of HIV at the hospital hosting the study using the same antibody test. At 6 weeks of age, infants were tested with HIV-1 DNA polymerase chain reaction (PCR) using T100 Thermal Cycler (Bio-Rad Laboratories Inc, UK). It was not possible to test the HIV-EU infants at 6 months of age because of the Kenya government guidelines on HIV-exposed infants which stipulates that the test should be done when the infants are 9 months old using an antibody test and confirmed with PCR. ${ }^{12}$ The HIV-EU infants' HIV tests were thus done at 9 months to confirm if the infants were still HIV-negative. Two HIV-EU infants tested positive at 9 months and were thus excluded from the study.

\section{Questionnaire data}

Using a structured questionnaire, data on socio-demographic and economic status, breastfeeding initiation within 1 hour of birth, exclusive breastfeeding, expression of breast milk, and counselling on breastfeeding were collected by recall at recruitment and at 6 months postpartum. Exclusive breastfeeding was defined as by WHO - feeding infants solely on breast milk 24hours preceding the interview. ${ }^{13}$ In this definition, ORS, drops, syrups (vitamin, minerals and medicines) are allowed (not considered as food or drink), while water is prohibited. The standard DHS question of recalling 24 hour infant intakes of the following foods was adopted: breast milk, plain water, sugar or glucose water, gripe water, sugar-salt-water solution, fruit juice, infant formula, tea infusion, milk, porridge and honey. ${ }^{14}$ 


\section{Determination of exclusive breastfeeding using DO technique}

Breast milk intake was measured when infants were 6 weeks and 6 months old using the DO technique developed by the International Atomic Energy Agency (IAEA). ${ }^{8}$ After the mother and the infant were weighed, their baseline (pre-dose) saliva samples were collected (day 0- $\mathrm{T}_{\mathrm{o}}$ samples) using a sterile cotton wool and syringe. Mothers were then given orally an accurately weighed dose $(30 \mathrm{~g})$ of deuterium oxide $\left({ }^{2} \mathrm{H}_{2} \mathrm{O}\right)$ through a straw. They were then instructed to feed the infant as usual and allowed to go home. At least $2 \mathrm{ml}$ of saliva samples were subsequently collected from both the mother and infant in $10 \mathrm{ml}$ polypropylene sterile tubes on days $1,2,3,4,13$ and 14 . Post-dose saliva collection was done at the same time of day as time for baseline saliva collection on day 0. All labelled sample tubes were separately secured in ziplock polythene bags and immediately frozen in $-20^{\circ} \mathrm{C}$ freezers. Samples were then transported in cool boxes for analysis at the Kenya Medical Research Institute (KEMRI) nutrition laboratory in Nairobi. Deuterium enrichment in mother/infant saliva over a 14-day period was measured against a standard in the Fourier Transform Infrared Spectrophotometer (FTIR 8400 Series, Shimadzu Corporation, Kyoto, Japan). Intake of water from other sources other than breast milk was then calculated using a spread sheet developed by IAEA. ${ }^{8}$ Amounts of non-human milk water consumed by infants being exclusively breastfed have been estimated. ${ }^{15}$ Intake of $<25$ g/day of non-human milk water per day was classified as EBF as recommended by IAEA. ${ }^{8}$ ORS also does contain water and would account for EBF by WHO definition but non-EBF by DO method. Nevertheless, in this present study none $(0 \%)$ of the infants were given ORS at both 6 weeks and 6 months postpartum. 


\section{Data entry, cleaning and analysis}

Data was entered in EPI6 and cleaned using MS Excel ${ }^{\mathrm{TM}}$ before being transferred to Statistical Package for Social Scientists (SPSS) version 20 for analysis. The Chi-squared test for 2 by 2 (for categorical variables) using Phi and Cramer's statistics and student's t-tests for independent samples (for continuous variables) were used to detect EBF differences between the two groups. Variables controlled for in the multivariable logistic analyses were infant sex, gravidity, maternal age, marital status and maternal education. Adjusted odds ratios were reported together with the respective p-values. Bias between the recall and DO determined EBF rates for HIV positive and negative mothers were computed as the number of the infants who were EBF by maternal recall minus those EBF by the DO technique divided by the total sample size.

\section{Ethical considerations}

Ethical approval was secured from Kenyatta National Hospital/University of Nairobi Ethics and Research Committee (KNH/UON/ERC). Only the mothers who gave written consent were enrolled and followed up.

\section{Result}

\section{Study profile}

Of the recruited 75 HIV-positive mothers with their infants, 7 were lost during the 2 weeks follow-up for DO technique measurements at 6 weeks postpartum (Figure 1). EBF analysis at 6 weeks postpartum was therefore done for $68 \mathrm{HIV}$-positive mothers. Four mother-infant dyads were not traced at 6 months postpartum follow-up. Two infants of HIV-positive mothers were themselves HIV-positive at 9 months. Therefore 62 HIV-positive mothers were interviewed for infant breastfeeding practices at 6 months after birth. Two mother-infant dyads did not complete 
the 2 weeks DO technique measurements at 6 months, leaving 60 mother-infant dyads for the EBF analysis. Out of the $68 \mathrm{HIV}$-negative mothers with their infants, 3 mother-infant dyads were lost during the 2 weeks follow-up for DO technique measurements at 6 weeks. Two mothers did not turn up at 6 months follow-up. Sixty-three mothers were therefore interviewed for the infant breastfeeding practices at 6 months after birth. One HIV-negative mother did not complete the 2 weeks follow-up for DO technique measurements at 6 months leaving 62 mother-infant dyads for the EBF analysis.

\section{General characteristics of the mother-infant dyads}

Table 1 shows the socio-demographic and economic characteristics of HIV-positive and negative mothers at enrolment. HIV-infected mothers were older $(\mathrm{p}=0.002)$ and less educated than the uninfected mothers $(\mathrm{p}=0.035)$. Most mothers had more than one child. A greater proportion of HIV-positive mothers had male infants than the HIV-negative mothers although this was not statistically significant $(\mathrm{p}=0.059)$.

About two-thirds of the study participants were dwelling in villages, the rest in towns. Most women participating in the study were married, in monogamous arrangements, living together with their partners and were Christians. Most mothers delivered in health facilities (hospital and clinics) and had vaginal deliveries for the infants under study. The economic status of the two groups was comparable as measured by their type of housing. Fifty seven percent of the HIVpositive mothers were on antiretroviral therapy (ART). 


\section{Infant feeding practices at 6 week and month 6 after birth}

Table 2 depicts the breastfeeding practices among mothers at 6 weeks and 6 months postpartum. Fifty two (52\%) of HIV-positive and 57.4\% HIV-negative mothers initiated breastfeeding within

the first hour after birth. The rates were close to the Kenyan national average of $61.3 \% .{ }^{15}$ Only a negligible proportion of the mothers expressed breast milk both at 6 weeks postpartum and 6 months of age and this confirmed the anecdotal evidence that this practice is uncommon in the study community. Most mothers in both groups had been counselled on breastfeeding during the perinatal period and between 6 weeks and 6 months postpartum. Although the proportion of HIV-positive mothers counselled perinatally was $10 \%$ more than for HIV-negative mothers, the difference was not statistically significant.

Table 3 shows that by 24 hour recall, a greater proportion of HIV-infected mothers exclusively breastfed than HIV-uninfected mothers both at 6 weeks [94.1\% versus $76.9 \%$, respectively (aOR: $7.81 ; 95 \% \mathrm{CI}: 1.9-31.6, \mathrm{p}=0.004)]$ and 6 months postpartum [75\% versus $59.7 \%$, respectively (aOR: 2.27; 95\% CI: 1.0-5.3, p=0.058)]. Infants who consumed $>25$ g/day of nonhuman milk water as indicated by DO technique were considered not to have been exclusively breastfed (Table 3). EBF rates at 6 weeks from the DO technique were borderline significantly different between the two groups: $23.5 \%$ and $13.8 \%$ for HIV-positive and negative mothers, respectively $(\mathrm{p}=0.059)$. At that point in time, $\mathrm{EBF}$ rates based on recall were 4 and 5 times higher than the rates based on DO for HIV-positive and negative mothers, respectively. The DO technique-determined EBF rates at 6 months postpartum were $43.3 \%$ and $24.2 \%$ for HIVpositive and HIV-negative mothers, respectively (aOR: 2.4; 95\% CI: 1.0-5.7, $\mathrm{p}=0.048$ ). 
Therefore at 6 months postpartum, the EBF rates based on recall were 1.7 and 2.5 times higher than rates based on DO technique for HIV-positive and HIV-negative mothers, respectively. At both 6 weeks and 6 months post-partum, the EBF rates as determined by the DO technique were lower than the global target of $50 \% .^{16}$

The bias between the two methods of EBF determination was more at 6 weeks than at 6 months for both HIV-positive and negative mothers (Table 3).

\section{Discussion}

In this study, we compared the EBF practice between HIV-positive and negative mothers using both recall and DO technique. A greater proportion of HIV-positive mothers exclusively breastfeed their infants as compared to their negative counterparts. The EBF rates determined by DO technique were lower than the maternally recalled EBF rates and it is apparent that irrespective of their HIV-status, mothers over-report EBF.

The observation that HIV-positive mothers exclusively breastfed more could be explained by the varying level of exposure to counselling and nutrition messages on infant feeding. Kenya fully subscribes to the WHO recommendations on infant feeding in the context of HIV.${ }^{17}$ In the present study, $10 \%$ more of the HIV-positive mothers than HIV-negative had been counselled on breastfeeding by 6 weeks postpartum. Further, counselling is more likely to impact on breastfeeding practice among the HIV-positive mothers due to the greater risk associated with mixed feeding. The knowledge among the HIV-positive mothers that the risk of transmission of the virus is lower if the infant is EBF could act as a motivation to EBF. Mothers are tested of HIV at pregnancy and most are aware of their HIV status by the time they give birth. In addition 
to the closer interaction with the health workers, HIV-positive mothers are often members of HIV-positive mother support groups where mothers share experiences and psychosocially support each other to care for their infants. This was the case for the study site where these support groups are active. The HIV-positive mothers are thus more exposed to health and nutrition messages than their HIV-negative counterparts. In South Africa before the WHO 2010 recommendations on HIV and infant feeding, EBF rates were lower among HIV-positive than HIV-negative mothers and replacement feeding was more acceptable among HIV-positive mothers. ${ }^{18,19}$ A decade prior to the advent of the WHO recommendations, a Kenyan study found by maternal recall that at 6 weeks postpartum only $30 \%$ of the HIV-positive mothers exclusively breastfed their HIV-negative infants. ${ }^{20}$ In the same study, none of the infants were being exclusively breastfed by the age of 4.5 months. It is apparent from the present study that the earlier tendency of the HIV-positive mothers not to breastfeed exclusively as compared to the HIV-negative mothers has been reversed. The provision of combination ART and antibiotics (septrin) to HIV-positive mothers also ensures they are less affected by opportunistic illnesses which may usually affect their ability to effectively care for and breastfeed their infants.

EBF rates found by this study at two points in time using the self-recall showed a different trend (with infants' ages) compared to other studies. In Kenya, EBF rates decline with the age of the infant. ${ }^{15,21}$ In the present study, the same was observed (irrespective of the maternal HIV status) for the recalled EBF rates. This has also been reported among the Tanzanian, Ethiopian and Nigerian mothers. ${ }^{22-24}$ Mothers may take time off from other activities to nurse their new-borns and this means more mother-infant contact around the time of birth and thus a high propensity for EBF. Later on when the infant is much older, they begin to engage more in other activities, 
reducing the mothers' nursing time. Furthermore, cultural practices may influence the introduction of complementary foods from an early age. Conversely, the EBF rates as determined by the DO technique increases between 6 weeks and 6 months postpartum. This study was conducted in a hospital set-up where breastfeeding mothers are closely followed up postpartum and counselled. There is a possibility that with these follow-ups and with time, mothers become more aware of the importance of EBF and practise it more. It has been shown that the number of visits by breastfeeding counsellors is associated with higher EBF rates. ${ }^{25}$ Among Ghanaian mothers, lactation counselling (and health worker-mother contact time) increased EBF rates up to 4 months postpartum. ${ }^{26}$ The counselling effect on EBF rates is more likely to be greater among the HIV-positive mothers, who are more exposed to counselling and are more closely followed up as compared to the HIV-negative mothers.

The EBF rates determined by DO technique were much lower than maternally recalled EBF rates regardless of maternal HIV status. Among Cameroonian mothers of infants averaging 2.5 months old, $82 \%$ of mothers over-reported EBF, which is comparable to the $75 \%$ over-reporting (DO technique verses self-recall). ${ }^{27}$ Over-reporting of the EBF was also shown among Botswana mothers when the two EBF determination techniques were compared. ${ }^{28}$ On the other hand, reported EBF practice by Bangladeshi mothers of infants averaging 14.3 weeks old was comparable to the objective DO technique among. ${ }^{29}$ Previous studies however did not focus on maternal HIV infection. This study is one of the first in comparing the reported verses DO determined EBF rates in the context of HIV. 
It has been estimated that non-breast milk water intake of EBF infants is $25+/-62 \mathrm{~g} / \mathrm{day} .{ }^{8}$. We used a single cut-off of $25 \mathrm{~g} /$ day with no consideration of the technical variability of the method and estimate, so may have misclassified some infants. In this present study, the results were also limited to the mothers who attended the post-natal clinic and not for the general population. In Kenya, $43 \%$ of women do not receive a postnatal check-up within the first six weeks after delivery. ${ }^{21}$ In addition, EBF did not depict the practice since birth, but at only two points in time.

\section{Conclusions}

In a Kenyan resource-poor setting, EBF rates by 6 months post-partum are low regardless of maternal HIV-status. HIV-positive mothers are however, more motivated to exclusively breastfeed compared to HIV-negative mothers. Validation of self-reported EBF practices with the low-cost, non-invasive deuterium oxide dilution technique is highly recommended to enable better understanding of reported EBF rates.

\section{Acknowledgements}

We are indebted and acknowledge the funding support from International Atomic Energy Agency (IAEA), the Nutricia Research Foundation and the National Commission of Science and Technology (NACOSTI) Kenya. The mothers and infants who agreed to participate in this research are also highly appreciated. The dedication of the project staff who worked under this project is hereby also acknowledged. 


\section{Funding}

This research was funded by the International Atomic Energy Agency (IAEA), The Nutricia Research Foundation and National Council for Science, Technology and Innovation (NACOSTI)

Kenya.

\section{Disclosure statement}

The authors declare that they had no competing interest before and during the research.

\section{References}

1. Vinther T and Helsing E. Breastfeeding, how to support success: A practical guide to health workers. World Health Organisation, Copenhagen, 1997.

2. WHO. Nutrient requirements for people living with HIV/AIDS. Report of a technical consultation. World Health Organization, Geneva, 2003.

3. UNICEF. HIV and infant feeding. Breastfeeding and HIV transmission; 2015 http://www.unicef.org/nutrition/index_24827.html [Accessed 16 July 2015].

4. WHO Guidelines for HIV and infant feeding. Principles and recommendations for infant feeding in the context of HIV and a summary of evidence. World Health Organization, Geneva, 2010.

5. Lutter CK, and Morrow AL. Promotion, and support and global trends in breastfeeding. Adv. Nutr 2013;4:213-219.

6. Engebretsen IMS, Wamani H, Karamagi C, et al. Low adherence to exclusive breastfeeding in Eastern Uganda: A community-based cross-sectional study comparing dietary recall since birth with 24-hour recall. BMC Pediatrics 2007;7:10. 
7. Kylberg AE, Hörnell A, Hofvander Y, et al. How exclusive is exclusive breastfeeding? A comparison of data since birth with current status data. Int. J. Epidemiol 2000;29:10411046.

8. IAEA. Stable isotope technique to assess intake of human milk in breastfed infants. International Atomic Energy Agency (IAEA), Vienna, 2010.

9. NAAC. HIV and AIDS county profiles. $2014 \mathrm{http}: / /$ http://www.nacc.or.ke/images/documents/KenyaCountyProfiles.pdf [Accessed 21st March 2016]

10. Kirkwood B, Sterne J. Medical Statistics. Blackwell Science Inc Malden, Massachusets, 2003.

11. Galpin L, Thakwalakwa C, Phuka J, et al. Breast milk intake is not reduced more by the introduction of energy dense complementary food than by typical infant porridge. J Nutr 2007; 137: 1828-1833

12. GOK. Guidelines for HIV Testing in Infants. Government of Kenya (GOK), Nairobi, 2012.

13. WHO. Indicators for Assessing Infant and Young Child Feeding Practices: Conclusions of a Consensus Meeting held 6-8 November 2007 in Washington D.C. USA. World Health Organization, Geneva, 2010.

14. GOK. Kenya Demographic and Health Survey (KDHS). Government of Kenya (GOK), Nairobi. 2014

15. Haisma H, Coward WA, Albernez E, et al. Breast milk and energy intake in exclusively, predominantly, and partially breast-fed infants. Eur J Clin Nutr 2003;57:1633- 42 
16. WHO and UNICEF, 2016. Global Nutrition Targets 2025: Breastfeeding policy brief. http://apps.who.int/iris/bitstream/10665/149022/1/WHO_NMH_NHD_14.7_eng.pdf?ua= 1 [Accessed 19 April 2016].

17. GOK. National Maternal, Infant and Young Child Nutrition Policy. Government of Nairobi, Kenya, 2013.

18. Bland RM, Kirsty EL, Coovadia HM, et al. Intervention to promote exclusive breastfeeding for the first 6 months of life in a high HIV prevalence area. AIDS 2008;22:883891.

19. Rollins N, Meda N, Becquet R, et al. Preventing postnatal transmission of HIV-1 through breast-feeding: modifying infant feeding practices J Acquir Immune Defic Syndr 2004; 35:188-195.

20. Sherry B, Embree JE, Mei Z, et al. Characteristics, care, feeding practices, and growth of cohorts of children born to HIV-1-seropositive and seronegative mothers in Nairobi, Kenya. TMIH.2000;5:678-686.

21. GOK. Kenya Demographic and Health Survey (KDHS). Government of Kenya (GOK), Nairobi, 2014.

22. Victor R, Baines SK, Agho KE, et al. Determinants of breastfeeding indicators among children less than 24 months of age in Tanzania: a secondary analysis of the 2010 Tanzania Demographic and Health Survey. BMJ Open 2013;3: e001529.

23. Agho1 KE, Dibley MJ, Odiase JI, et al. Determinants of exclusive breastfeeding in Nigeria. BMC Pregnancy Childbirth 2011; 11. http://www.biomedcentral.com/14712393/11/2 [Accessed 19th January 2016] 
24. Setegn T, Belachew T, Gerbaba M, et al. Factors associated with exclusive breastfeeding practices among mothers in Goba district, south east Ethiopia: a cross-sectional study. Int Breastfeed J 2012;7:17.

25. Morrow AL, Guerrero ML, Shults J, et al. Efficacy of home-based peer counselling to promote exclusive breastfeeding: a randomised controlled trial. Lancet 1999;353:12261231

26. Aidam BA, Pe’ rez-Escamilla R, Lartey A. Lactation counselling increases exclusive breast feeding rates in Ghana. J. Nutr 2005;135:1691-1695

27. Medoua GN, Nana ECS, Ndzana ACA, Makamto CS, Etame LS, Rikong HA and Oyono JLE. Breastfeeding practices of Cameroonian mothers determined by dietary recall since birth and the dose-to-the-mother deuterium-oxide turnover technique. Matern Child Nutr 2012;8:330-339

28. Motswagole BS, Mongwaketse T, Kobue-Lekalake R, et al. Application of the deuterium-oxide dose-to-mother technique to determine the exclusivity of breastfeeding in women in Kanye, Botswana. S Afr J Clin Nutr 2015;28:128-133

29. Moore SE, Prentice AM, Coward WA, et al. Use of stable-isotope techniques to validate infant feeding practices reported by Bangladeshi women receiving breastfeeding counselling. Am J Clin Nutr 2007;85:1075-82. 